\title{
Rethinking general anesthesia for cesarean section
}

\author{
Yoshihisa Fujita $^{1,2} \cdot$ Masatsugu Tsuge ${ }^{1}$
}

Received: 24 March 2016 / Accepted: 29 April 2016 / Published online: 19 May 2016

(C) Japanese Society of Anesthesiologists 2016

Keywords Cesarean section · Rapid-sequence induction

\section{To the Editor:}

We read with interest the review article by Sumikura et al. [1] on current consensus guidelines for general anesthetic management of patients undergoing cesarean section. We learned in the review that a "sleeping baby" may be an acceptable consequence of anesthetic management when neonatologists are fully prepared to care for the newborn in the operating room itself. However, we believe that the conclusion drawn by the authors is not well discussed and that the underlying reasoning is not sound. The authors state "However, due to the reduction in education opportunities regarding general anesthesia, in addition to the increase in older and obese parturients, the safety of general anesthesia may decline again". Regional anesthesia is currently the preferred method (vs. general anesthesia) for cesarean section; however, to the best of our knowledge there has not been a recent decline in educational opportunities regarding general anesthesia. This point was not discussed by the authors in their review. In addition, this statement by the authors should be underpinned by at least two references, with the first showing that the prevalence of older and obese pregnant women has increased in the last decade and the second showing that such a small increase in

Yoshihisa Fujita

fujitay@med.kawasaki-ma.ac.jp

1 Department of Anesthesiology and ICM, Kawasaki Medical School Hospital, 577 Matsushima, Kurashiki, Okayama 7010192, Japan

2 Present Address: Department of Anesthesia, Iwaki Kyoritsu General Hospital, Iwaki, Japan mean age could lead to an increased risk for general anesthesia. Although age is known to be related to mortality due to anesthesia in general, the age group of 19-49 years is a very safe patient group [2]. We do not believe an increase in the mean age of pregnant women as mentioned by the authors results in an increase in mortality.

We also found a typographical error on the last page of the article: "as soon as soon as possible". We therefore encourage all participants at all levels of the publication process to excercise care in maintaining the integrity and quality of the Journal of Anesthesia.

\section{Compliance with ethical standards}

Conflict of interest The authors report no financial support or potential conflicts of interest for this work.

\section{References}

1. Sumikura H, Niwa H, Sato M, Nakamoto T, Asai T, Hagihira S. Rethinking general anesthesia for cesarean section. J Anesth. 2016;30:268-73.

2. Whitlock EL, Feiner JR, Chen LL. Perioperative mortality, 2010 to 2014: a retrospective cohort study using the National Anesthesia Clinical Outcomes Registry. Anesthesiology. 2015;123:1312-21. 